\title{
Hearing Rehabilitation with Combined Electroacoustic Stimulation and Ossiculoplasty
}

\author{
Jeon Mi Lee ${ }^{1}$, Gina $\mathrm{Na}^{2}$, and Jae Young $\mathrm{Choi}^{2}$ \\ 1Department of Otorhinolaryngology, Ilsan Paik Hospital, Inje University College of Medicine, Goyang, Korea \\ ${ }^{2}$ Department of Otorhinolaryngology, Yonsei University College of Medicine, Seoul, Korea
}

\author{
Received April 3,2020 \\ Revised June 24,2020 \\ Accepted July 24, 2020
Address for correspondence
Jae Young Choi, $\mathrm{MD}, \mathrm{PhD}$
Department of Otorhinolaryngology,
Yonsei University
College of Medicine,
50-1 Yonsei-ro, Seodaemun-gu,
Seoul 03722, Korea
Tel $+82-2-2228-3620$
Fax +82-2-393-0580
E-mail jychoi@yuhs.ac

\begin{abstract}
We report a case of hearing rehabilitation following combined cochlear implantation and ossiculoplasty. A 71-year-old patient visited the clinic for right-sided mixed hearing loss. We targeted neural and conductive components, performing two different operations simultaneously. At two months post-operative, the patient showed satisfactory results with respect to hearing threshold and speech comprehension. Our experience suggests that careful evaluation of patients and consideration of the diverse array of available treatment strategies can be used to provide personalized rehabilitation with maximal effectiveness. To the best of our knowledge, this is the first report to take such an integrated approach to treat hearing impairment and is thus likely to have clinical importance for otologists.
\end{abstract}

J Audiol Otol 2021;25(2):110-114

KEY WORDS: Hearing impaired rehabilitation · Cochlear implants · Ossicular replacement . Mixed hearing loss.

\section{Introduction}

Hearing impairment takes various forms and can be due to a number of causes. Individuals with hearing impairment have greater levels of anxiety and depression than the general population, which reflects the important role of hearing in quality of life [1]. Diverse methods have been developed to overcome hearing impairment, ranging from conventional hearing aids to surgical approaches such as ossiculoplasty and cochlear implantation (CI) for patients with impaired conductive and sensorineural hearing, respectively. Moreover, newly developed techniques have increased the possibility for rehabilitation using implantable bone conduction hearing aids, middle ear implantation, or auditory brainstem implantation and are producing good results [2-4]. On the other hand, these techniques target different steps in the auditory pathway, and thus their applicable indications and target sound frequencies are varied and limited.

Here, we present a case of hearing rehabilitation performed

This is an Open Access article distributed under the terms of the Creative Commons Attribution Non-Commercial License (https://creativecommons.org/licenses/by-nc/4.0/) which permits unrestricted non-commercial use, distribution, and reproduction in any medium, provided the original work is properly cited. by combining electroacoustic stimulation (EAS) and ossiculoplasty to maximize hearing gain. This study was approved by the Institutional Review Board of the Yonsei University College of Medicine (4-2020-0509). To the best of our knowledge, this is the first report of this strategy. Thus, the case presented here should be of clinical importance for otologists.

\section{Case Report}

A 71-year-old female visited our clinic presenting with bilateral hearing loss. Her medical history included chronic otitis media in both ears since childhood. The left ear was treated surgically 30 years prior, and her hearing on that side had been absent since then. Conversely, the patient's right side was treated surgically 20 years prior, and she had not had a relapse in hearing loss until the current episode. Specifically, following surgery on her right ear for chronic otitis media, she had depended on her right ear for hearing with a conventional hearing aid, but her hearing had recently progressively decreased.

Both eardrums had well tympanized tympanic membranes and canal wall down mastoidectomy status (Fig. 1A). Puretone average (PTA) revealed profound hearing loss in the left ear, and the speech test could not be conducted. PTA revealed 
mixed hearing loss in the right ear, with an average air-bone gap of $25 \mathrm{~dB}$ and an average air conduction threshold of over $80 \mathrm{~dB}$. The word recognition score (WRS) was $45 \%$ at the most comfortable level (MCL) of $98 \mathrm{~dB}$. PTA with a hearing aid was $51 \mathrm{~dB}$, and low frequencies from 250 hertz $(\mathrm{Hz})$ to 1,000 $\mathrm{Hz}$ showed a serviceable hearing threshold specifically (Fig. 1B). The WRS also increased to $68 \%$ with the MCL of $60 \mathrm{~dB}$. The computed tomography scan indicated canal wall down mastoidectomy status on both sides. The ossicles were absent, but the oval window was patent in the right ear (Fig. 1C). The middle ear cavity was well aerated through a widely opened Eustachian tube (Fig. 1D). Based on these findings, the patient was scheduled to undergo combined CI and ossiculoplasty for both sensorineural and conductive components.

Under general anesthesia, the mastoid cavity was exposed via a previous retroauricular incision. The canal wall downed mastoid cavity was trimmed to expose the round window, and the bony overhang of the round window was drilled to provide a better approach. A Bony bed was drilled at the temporal area, and the receiver-stimulator was inserted and sufficiently anchored with an overtie. Then, the oval window and stapes foot plate were identified. The stapes footplate was found to be well mobilized, and a total ossicular replacement prosthesis (TORP) was placed on the stapes footplate. Before creating the opening to the round window, systemic steroid was intravenously injected and the facial recess was also filled with a topical steroid. An electrode (Flex 24, MED-EL, Innsbruck, Austria) was then inserted into the scala tympani via the round window. This procedure was performed carefully and slowly so as not to damage the cochlea. Harvested tragal
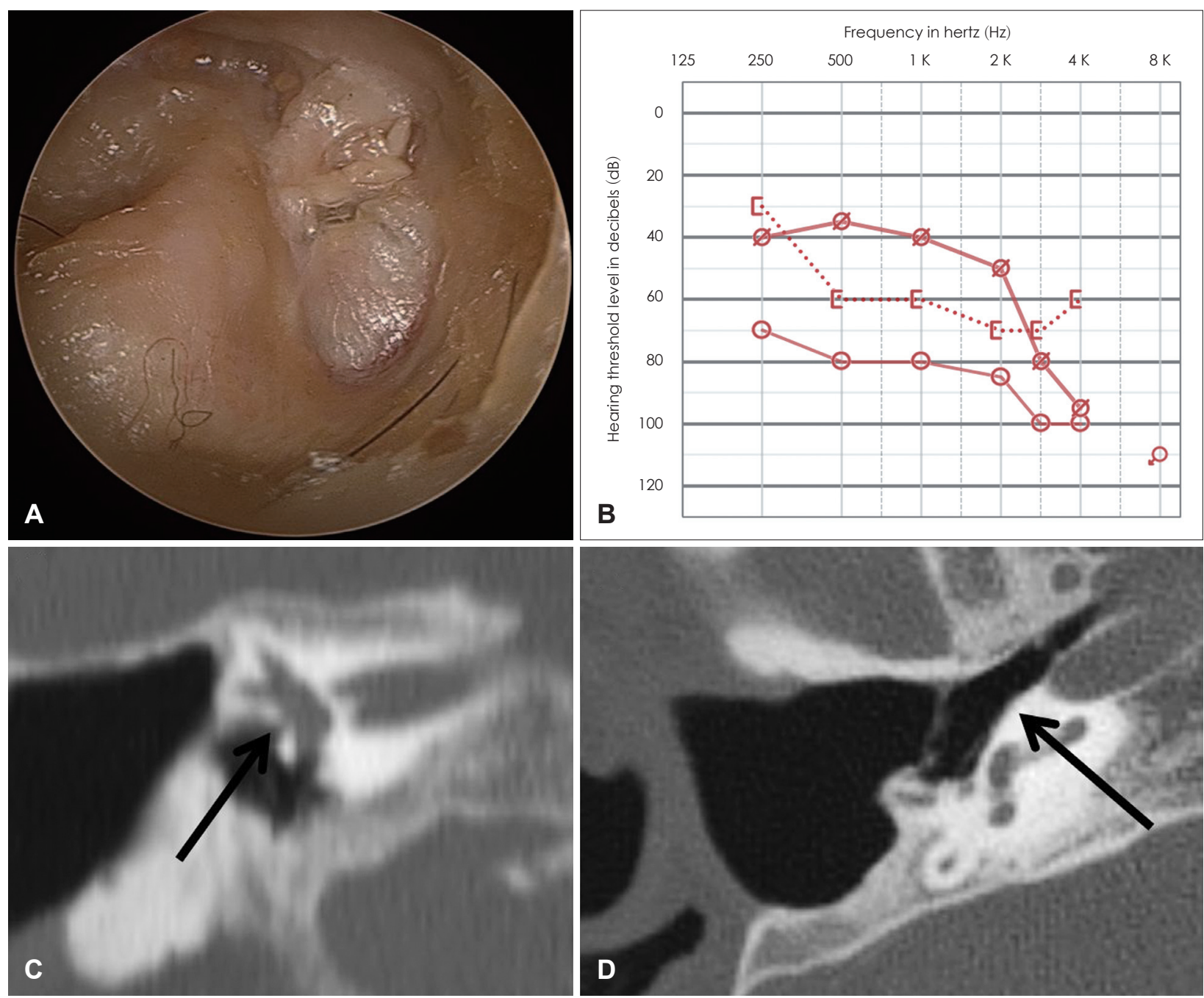

Fig. 1. Initial findings of the right ear. (A) The mastoid cavity was canal wall downed, and the tympanic membrane was well tympanized. (B) Pure-tone average showed mixed hearing loss in the right ear, with an average air-bone gap of $25 \mathrm{~dB}$ and an average air conduction threshold higher than $80 \mathrm{~dB}$. Low frequencies from $250 \mathrm{hertz}(\mathrm{Hz})$ to $1,000 \mathrm{~Hz}$ showed a serviceable hearing threshold with a hearing aid. (C) Computed tomography scan showing canal wall downed mastoidectomy status. The oval window was well opened (arrow), but the ossicles were not apparent. (D) The middle ear cavity was well aerated through a widely opened Eustachian tube (arrow). 
cartilage was interpositioned between the TORP and tympanic membrane (Fig. 2), and the remaining electrode was covered with bone dust as a precaution against unintended exposure of the middle ear cavity. The ear canal was not closed so as to preserve the air conduction. The patient was discharged on the second postoperative day without any complications.

The speech processor (Sonnet, MED-EL, Innsbruck, Austria) was turned on four weeks after surgery, and post-operative evaluation was performed after two months. Postoperative bone conduction was well preserved with decreased airbone gap. The patient was capable of serviceable hearing via acoustic stimulus with frequencies less than $500 \mathrm{~Hz}$ and electrical stimulus at higher frequencies (Fig. 3). Lastly, speech comprehension tests indicated that the patient's ability to comprehend sentences had increased from $48 \%$ to $88 \%$. The patient was satisfied with the results of combined EAS and ossiculoplasty, and a hearing test conducted 2 year after surgery showed no decline of hearing.

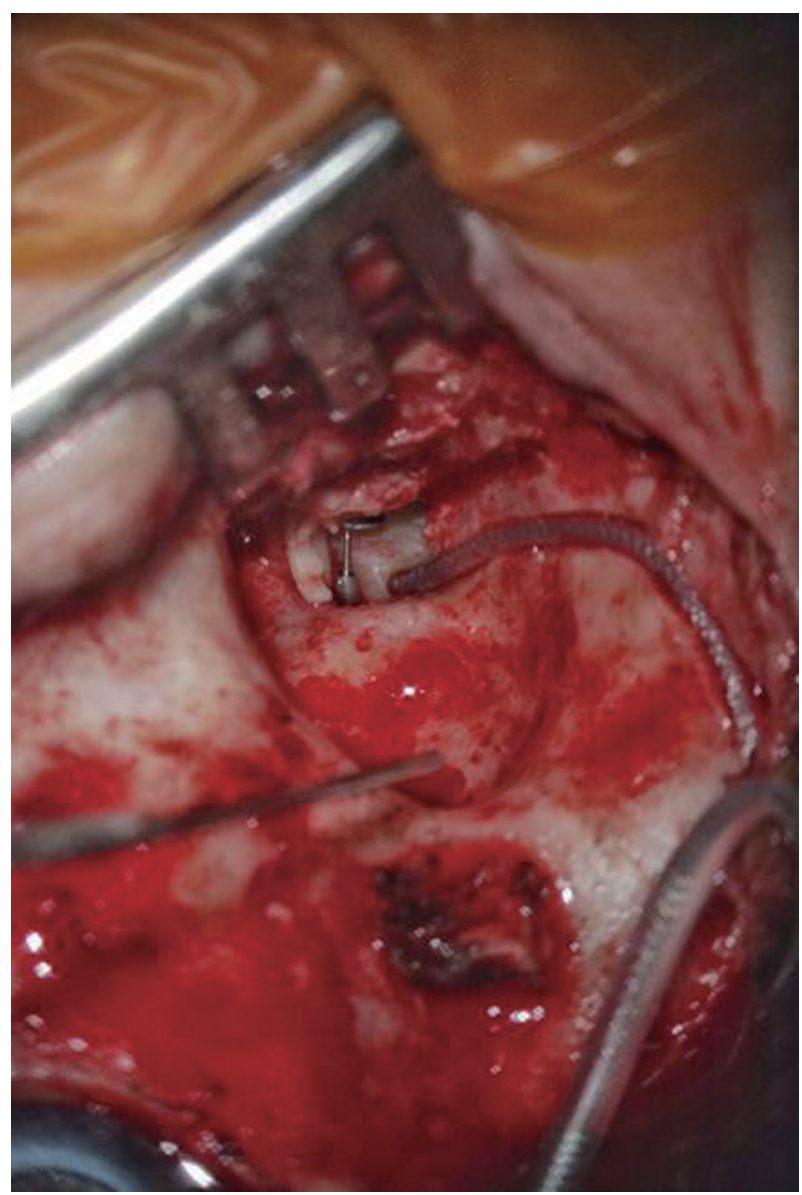

Fig. 2. Electrode inserted through the round window. The total ossicular replacement prosthesis was placed on the stapes footplate.

\section{Discussion}

This is the first case report of hearing rehabilitation using combined EAS and ossiculoplasty in patients with severe mixed hearing loss. For the patients with mixed hearing loss, various methods can be applied; bone-anchored hearing devices, conventional hearing aid after ossiculoplasty or Vibrant Soundbridge (VSB), the active middle ear implant devices. In this case, bone-anchored hearing device was not indicated since the bone conduction hearing thresholds were hardly serviceable, and the patient had no hearing in the opposite ear. Wearing a conventional hearing aid after ossiculoplasty was considered to be not as effective as using VSB. While ossiculoplasty mainly improves hearing in low to mid-range frequencies, VSB is effective for improving hearing in mid to high-frequencies which enables better comprehension in daily conversation [5]. A previous report compared the hearing outcome between conventional hearing aids after ossiculoplasty and vibroplasty in patients with mixed hearing loss, and vibroplasty showed superior results over conventional hearing aids after ossiculoplasty [6]. For patients with conductive or mixed hearing loss, the bone conduction should not be better than 45-65 dB within the frequencies 500 to $4,000 \mathrm{~Hz}$ as recommended by the company. There is not a speech discrimination criterion for the VSB implantation, but the outcome depends on this parameter. Although patients with a poor preoperative discrimination score of less than $40 \%$ (unaided) may show improvement with VSB, patients with discrimination score of $50-70 \%$ and higher are

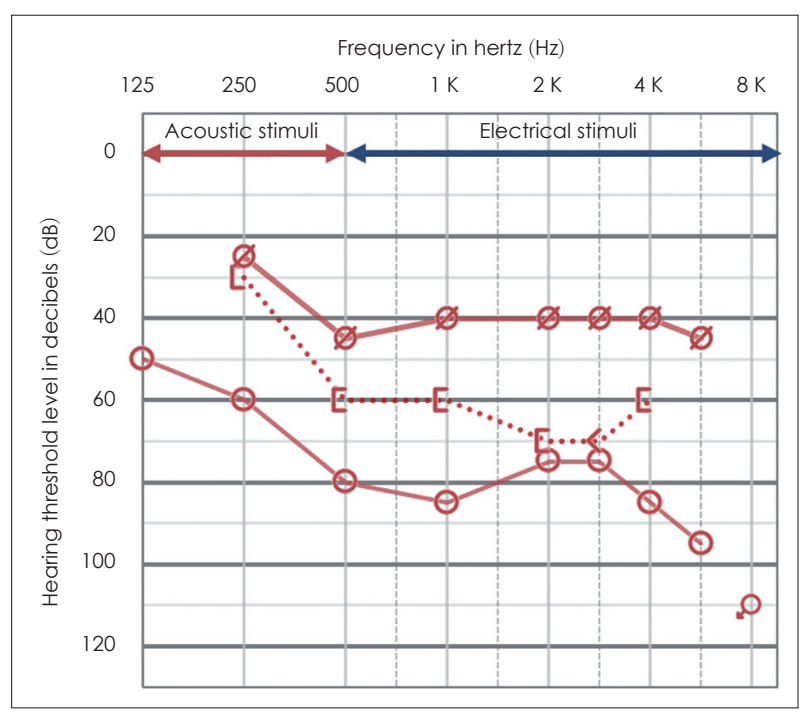

Fig. 3. Postoperative two-month pure-tone average. Bone conduction hearing was well preserved, and the air-bone conduction gap was decreased after combined electroacoustic stimulation and ossiculoplasty. The patient was capable of perceiving acoustic stimuli at frequencies lower than $500 \mathrm{~Hz}$ and electrical stimuli greater than $500 \mathrm{~Hz}$, demonstraing serviceable hearing. 
likely better candidates and long-term performance is likely to be better [7]. Therefore, considering the patient's preoperative hearing ability and the characteristics of each hearing rehabilitation methods, combined EAS and ossiculoplasty was the method of choice in this case, rather than the other methods available.

Electric acoustic stimulation (EAS) is the combination of acoustic stimulation via hearing aid and electrical stimulation via $\mathrm{CI}$ in the implanted ear. EAS is indicated for patients with residual hearing at low frequencies and severe or profound hearing loss at high frequencies. EAS recipients experience several benefits in hearing performance compared to recipients who rely on electrical stimulation alone with regard to pitch perception, speech perception, background noise, and music appreciation; however, the specific interactions between electrical and acoustic stimuli have not yet been clearly identified. Many attempts have been made to determine clinical information such as the proper range of spectral overlap of two stimuli and the minimum qualification for hearing ability. Although limited in its findings, a study by Ting Zhang [8] reported that the ability to comprehend acoustic information below $250 \mathrm{~Hz}$ significantly improves performance in EAS recipients, even though it affords little to no improvement in speech intelligibility on its own. The mechanism of this synergy is unknown; however, electrode stimulation is known to discern differences in pitch for frequencies up to about 300 Hz. Because $300 \mathrm{~Hz}$ represents the upper boundary of the temporal code, the synergism might be due to the temporal coding system [9]. Consistently, adding acoustic information above $250 \mathrm{~Hz}$ does not provide significant clinical benefits [8]. Therefore, hearing preservation, especially below 250 $\mathrm{Hz}$, is an aspect of EAS that can be considered during rehabilitation. In the present case, the acoustic energy below 250 $\mathrm{Hz}$ improved as compared to that before the surgery by performing combined ossiculoplasty in rehabilitating the hearing using EAS. This improved acoustic energy is expected to enhance the performance in EAS recipients.

Many factors influence the results of EAS, including use of a flexible electrode and steroids applied either topically or systemically; however, soft surgical techniques are the most important for preserving hearing. In the present case, we utilized gentle drilling on the bony overhang of the round window, and both systemic and topical steroid were applied. Trauma to structures of the lateral cochlear wall is one of the most commonly reported types of insertional damage and is a significant type of injury related to hearing preservation [10]. For this reason, we slowly and gently inserted a flexible electrode via the round window along the lateral wall. These efforts enabled the complete preservation of bone conduction, which lead to successful hearing rehabilitation with EAS.

In addition to the application of atraumatic surgical techniques, partial electrode insertion has been suggested in the past for preserving hearing, and short- to medium- length electrodes have been used for EAS applications. On the contrary to this, Mick, et al. [11] reported preservation of hearing following full insertion of the electrode, with the idea that the risk of re-implantation for longer electrodes could be decreased in cases where hearing was not initially preserved. Although such full-length electrode insertion is possible, the electrode is typically implanted to depths ranging from $8-21 \mathrm{~mm}$ due to anatomical and design limitations, corresponding to cochlear frequencies no lower than $500-1,500 \mathrm{~Hz}$. This limitation results in a tonotopic mismatch between the sound frequencies analyzed by the processor and the actual auditory nerve fibers stimulated electrically in the cochlea, which can either overlap or result in a gap in the auditory spectrum. This has been a significant concern since the concept of EAS was first introduced. While the mechanism by which two stimuli are combined has not been identified, there are some interesting reports that the presence of holes in the auditory spectrum does not appear to change one's ability to recognize speech [12]. Similarly, spectral overlap does not affect speech perception regardless of whether the overlapping spectrum is wide or narrow [8]. Nevertheless, pitch perception provided by electrical stimulation at a particular cochlear location can change over time in EAS recipients compared to an acoustic reference [13]. Thus, the spectral range of speech recognition maintains continuity due to adjustment to these changes.

Based on the above discussion, one might assume that neural plasticity changes do not occur at the level of the cochlear organ, but rather at the level of the auditory cortex. Specifically, the auditory cortex permits early spectral mismatch and gradually controls the peripheral organs, allowing for tonotopicity of the cochlear nerve and spectrum rearrangement. This rearrangement takes time and is affected by variables such as age, frequency of hearing aid use, and adequacy of amplification. Since pitch perception provided by electrical stimulation changes in order to maintain continuity, rearrangement might take longer when the mismatch gap is larger [13]. However, time to rearrangement does not mean time to adaption, as adaptation and permission start in the auditory cortex in early stages and proceed to the peripheral organs. Ultimately, further studies are needed to clarify the mechanisms of acoustic rearrangement and adaptation.

Ossiculoplasty allows for recovery of not only the continuity of the ossicular chain, but also normal middle ear pressure gain, which can lead to significant improvements in hearing, especially for low to mid-range frequencies. Indeed in the pres- 
ent case, the aided hearing thresholds in frequencies less than $500 \mathrm{~Hz}$ showed remarkable hearing improvement compared to preoperation. This can be an advantageous outcome in patients planning on undergoing EAS surgery, as it saves the low frequencies and stimulates only high frequencies. However, despite this advantage for hearing, we considered only EAS in the present case at the first time because of the possibility of TORP extrusion. Because the patient suffered from chronic otitis media, there was a significant chance for retraction of the middle ear cavity and extrusion of the prosthesis in the middle ear cavity. To prevent the extrusion of the prosthesis, we performed preoperative CT to confirm that the Eustachian tube had a normal shape, and its function was confirmed based on aeration of the middle ear cavity. Lastly, interpositioned cartilage could provide good protection from extrusion as well.

Initially, the patient did not experience a clear benefit from initial use of the EAS; as a consequence did not try to use the implant. Nevertheless, we encouraged her to use the implant, to which she eventually became accustomed. While it is known that patients who have experienced deafness for a shorter period of time prior to implantation exhibit better and faster results when using EAS [14], it is also more difficult for these patients to adapt to electrical sounds [15]. Use of implants for post-lingual hearing loss, mainly due to aging, is now increasing. For the best implantation results, many factors in addition to hearing testing, including patient age, duration of deafness, and auditory experience prior to implantation, should be considered. Also, the encourage from the clinicians would be helpful for the patients to be accustomed to new conditions.

Many strategies have been developed for hearing rehabilitation, and each method has its own indications and limitations. Here, we presented a case of hearing rehabilitation by combining EAS and ossiculoplasty in a single stage in order to maximize hearing gain. Our experience suggests that careful evaluation of the patient and consideration of available strategies can be used to provide personalized maximal hearing rehabilitation.

\section{Acknowledgments}

This work was supported by the National Research Foundation of Korea (NRF) grant funded by the Korea government (MSIT) (No. 2019R1C1C1006341).

\section{Conflicts of interest}

The authors have no financial conflicts of interest.

\section{Author Contributions}

Conceptualization: Jae Young Choi. Data curation: Jeon Mi Lee and Gina Na. Funding acquisition: Jeon Mi Lee. Supervision: Jae
Young Choi. Validation: Jae Young Choi. Visualization: Jae Young Choi. Writing — original draft: Jeon Mi Lee. Writing — review \& editing: Jae Young Choi. Approval of final manuscript: all authors.

\section{ORCID iDs}

Jeon Mi Lee

Gina Na

Jae Young Choi https://orcid.org/0000-0003-2793-9045

https://orcid.org/0000-0001-8111-2124

https://orcid.org/0000-0001-9493-3458

\section{REFERENCES}

1) Carlsson PI, Hjaldahl J, Magnuson A, Ternevall E, Edén M, Skagerstrand $\AA$, et al. Severe to profound hearing impairment: quality of life, psychosocial consequences and audiological rehabilitation. Disabil Rehabil 2015;37:1849-56.

2) Reinfeldt $S$, Håkansson B, Taghavi H, Fredén Jansson KJ, EegOlofsson M. The bone conduction implant: clinical results of the first six patients. Int J Audiol 2015;54:408-16.

3) Zwartenkot JW, Hashemi J, Cremers CW, Mulder JJ, Snik AF. Active middle ear implantation for patients with sensorineural hearing loss and external otitis: long-term outcome in patient satisfaction. Otol Neurotol 2013;34:855-61.

4) Goffi-Gomez MV, Magalhães AT, Brito Neto R, Tsuji RK, Gomes Mde Q, Bento RF. Auditory brainstem implant outcomes and MAP parameters: report of experiences in adults and children. Int J Pediatr Otorhinolaryngol 2012;76:257-64.

5) Lee HJ, Lee JM, Choi JY, Jung J. Evaluation of maximal speech intelligibility with vibrant soundbridge in patients with sensorineural hearing loss. Otol Neurotol 2017;38:1246-50.

6) Lee JM, Jung J, Moon IS, Kim SH, Choi JY. Benefits of active middle ear implants in mixed hearing loss: stapes versus round window. Laryngoscope 2017;127:1435-41.

7) Baumgartner WD, Böheim K, Hagen R, Müller J, Lenarz T, Reiss S, et al. The vibrant soundbridge for conductive and mixed hearing losses: European multicenter study results. Adv Otorhinolaryngol 2010; 69:38-50.

8) Zhang T, Spahr AJ, Dorman MF. Frequency overlap between electric and acoustic stimulation and speech-perception benefit in patients with combined electric and acoustic stimulation. Ear Hear 2010;31: 195-201.

9) Talbot KN, Hartley DE. Combined electro-acoustic stimulation: a beneficial union? Clin Otolaryngol 2008;33:536-45.

10) Roland PS, Wright CG. Surgical aspects of cochlear implantation: mechanisms of insertional trauma. Adv Otorhinolaryngol 2006;64:1130.

11) Mick P, Amoodi H, Shipp D, Friesen L, Symons S, Lin V, et al. Hearing preservation with full insertion of the FLEXsoft electrode. Otol Neurotol 2014;35:e40-4.

12) Gantz BJ, Turner CW. Combining acoustic and electrical hearing. Laryngoscope 2003;113:1726-30.

13) Reiss LA, Turner CW, Karsten SA, Gantz BJ. Plasticity in human pitch perception induced by tonotopically mismatched electro-acoustic stimulation. Neuroscience 2014;256:43-52.

14) Arndt S, Prosse S, Laszig R, Wesarg T, Aschendorff A, Hassepass F. Cochlear implantation in children with single-sided deafness: does aetiology and duration of deafness matter? Audiol Neurootol 2015;20 Suppl 1:21-30.

15) Büchsenschütz K, Arnolds J, Bagus H, Fahimi F, Günnicker M, Lang $\mathrm{S}$, et al. [Surgical risk profile and audiological outcome in the elderly after cochlea-implantation.]. Laryngorhinootologie 2015;94:670-5. 